\title{
Cardiovascular Disease and Metabolic Syndrome in Infertility
}

\author{
Lozano-Hernández $\mathbf{R}^{*}$ \\ Center of Infertility and Gynecological Diseases, University of Los Andes, Venezuela
}

*Corresponding author: Ricardo Lozano-Hernández, Center of Infertility and Gynecological Diseases (CEDIEG) “Dr. Giovanny Vivas-Acevedo”, University of Los Andes, Mérida, Venezuela

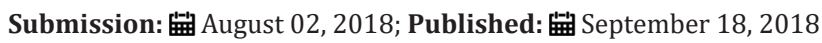

\begin{abstract}
Dyslipidemia, hypertension and other symptoms are part of the well-known metabolic syndrome (MS). MS is extended well beyond the cardiovascular system. In infertile men the androgen deficiency is associated with increased triglycerides (TGs), total cholesterol (TC), and low-density lipoprotein cholesterol (LDL-C). Increase T levels are associated with lower risk in men and with higher risk in women. It is necessary to keep in mind that cardiovascular diseases can be influenced by sex hormones and other types of hormones. Testosterone is a pleiotropic hormone that plays an important role in the human body. Through its conversion to E2, T affects bone health, including bone density. BMI has been correlated with the value of systolic blood pressure, ratio LH/FSH and T, and it was inversely correlated with the hormones FSH and PRL in infertile woman with polycystic ovary. The influence of many hormones on the cardiovascular function also depends on act directly through specific receptors in heart or vessel wall cells, whereas some act indirectly - stimulating other neuroendocrine factors. The majority of those hormones play an important role in the pathogenesis of cardiovascular diseases, which can result in the development of new medicines.
\end{abstract}

In conclusion, cardiovascular diseases may be the main or associated cause of reproductive failure in infertile couples: however, the interactions that exist between the cardiovascular system and the endocrine reproductive system must be focused on each patient individually.

Keywords: Dyslipidemia; Hypertension; Metabolic syndrome; Infertility

Abbreviations: BMI: Body Mass Index; DHEA-S: Dehydroepiandrosterone-Sulphate; E2: Estradiol; FT: Free Testosterone; HDL-C: High-Density Lipoprotein Cholesterol; HOMA-IR: Homeostasis Model Assessment of Insulin Resistance; LDL-C: Low-Density Lipoprotein Cholesterol; PCO: Polycystic Ovary; PRL: Prolactine; T: Testosterone; TC: Total Cholesterol; TGs: Triglycerides

\section{Introduction}

Dyslipidemia and hypertension are frequent symptoms in infertile couples. These symptoms in compilation with other as central obesity and insulin resistance are part of the well-known metabolic syndrome (MS). Initially MS was used to predict cardiovascular disease, it is now clear that the molecular and physiologic abnormalities seen in metabolic syndrome extend well beyond the cardiovascular system. Growing evidence has linked MS and its individual symptoms to the increasing prevalence of infertility [1]. Hypertension and dyslipidemia increase the risk of long-term cardiovascular disease in type 2 diabetes [2], which are disproportionally more harmful in low- and middle-income countries than in high-income countries [3].

The relationship between androgen deficiency and atherosclerosis is controversial. Studies suggest that androgen deficiency is associated with increased triglycerides (TGs), total cholesterol (TC), and low-density lipoprotein cholesterol (LDL-C) in infertile men. The androgen therapy has been associated with increased levels of high-density lipoprotein cholesterol HDL-C and may improve reverse cholesterol transport [4]. The incidence of obesity (18\%), overweight (30.2\%), diabetes mellitus (4.7\%), glucose intolerance (15\%), hypertension (26\%) and dysplipidemia (65\%) was observed en infertile men. The dyslipidemia was isolated hypercholesterolemia, isolated triglyceridemia or both. In them a positive correlation between estradiol (E2) and follicle-stimulating hormone (FSH) was observed when estradiol levels exceed $50 \mathrm{pg} / \mathrm{mL}$ [5]. In men with coronary artery disease Gensini score (to reflect the extent and severity of coronary atherosclerosis) didn't show correlation neither with the number of involved segments nor with the androgen levels. TGs, TC and LDL-C levels also had no correlation with testosterone (T), free-testosterone (FT) nor dehydroepiandrosterone-sulphate (DHEA-S). However, FT showed negative correlation with lipoprotein (a) and C-reactive protein [6].

Another heart disease recently associated with hormonal changes is atrial fibrillation, which is the most common serious abnormal heart rhythm, and a frequent cause of ischaemic stroke. Increase $\mathrm{T}$ serum levels were associated with lower risk in men and 
with higher risk in women. So that low testosterone levels are associated with increased risk of future atrial fibrillation and/or ischaemic stroke in men, while they are protective in women [7].

Low T serum levels are observed among men with Type 2 Diabetes Mellitus and are inversely related to insulin resistance. $\mathrm{T}$ in diabetic men has been inversely related to body mass index (BMI), waist circumference, and glucose tolerance, pre and post insulinemia and homeostasis model assessment of insulin resistance (HOMA-IR) [8]. BMI has positive relation with the value of systolic blood pressure, ratio LH/FSH and T, and negative correlation with FSH and PRL in infertile woman with polycystic ovary (PCO). Last patients with BMI $>24 \mathrm{~kg} / \mathrm{m}^{2}$ showed higher systolic pressure and DHEA- $S$ and post insulinemia levels in comparison with a $<24 \mathrm{~kg} /$ $\mathrm{m}^{2}$ group [9]. Other associations have been observed in males with hypertension, the link between insulin sensitivity and hypothalamic-pituitary-gonadal axis is maintained along the entire spectrum of glucose tolerance [8]. Obesity can be related to cardiovascular, metabolic problems, prostate dysfunction, and declining on sperm parameters in morbid obesity. Oxidative stress, hormonal and metabolic changes increase as adiposity progresses in an individual, so we may expect infertility, prostate pathologies could be avoided if hyperadiposity in infertile men is controlled opportunely [10].

It is necessary to keep in mind that cardiovascular diseases can be influenced by sex hormones, such as other types of hormones. $\mathrm{T}$ is a pleiotropic hormone that plays an important role in the human body. Through its conversion to E2, T affects bone health, including bone density. There has been a renewed interest in the systemic role of $\mathrm{T}$ in pain, well-being, and cardiovascular function in women and men alike. It's necessary to revisit the clinical role of $\mathrm{T}$ given its potential for applications to treat mood, cognitive health, and other illnesses, and its anabolic role in bone and muscle; it must also be treated carefully when taking into account the risks of its excessive use [11]. In native men from high altitudes it has been observed that higher serum $\mathrm{T}$ levels are associated with excessive erythrocytosis and with lower sperm motility [12]. Endocrine regulation in cardio-vascular system (CVS) may occur in many ways. Apart from hormones usually connected with CVS regulation, other more can act on it. A few of these act directly through specific receptors in heart or vessel wall cells, whereas some act indirectly - stimulating other neuroendocrine factors. Additionally, novel mechanisms of signal transduction have been discovered for steroid and thyroid hormones, which are independent of gene transcription regulation and are -known as "nongenomic". It's difficult to exclude other type of hormones such as urotensin II, endothelins, angiotensin II, catecholamines, aldosterone, antidiuretic hormone, glucocorticosteroids, thyroid hormones, growth hormone and leptin that increase blood pressure. Also the decrease of hypotensive substances as natriuretic peptides, the calcitonin gene-related peptide (CGRP) family, angiotensin 1-7, substance P, neurokinin A, ghrelin, Parathyroid hormone-related protein (PTHrP) and oxytocin which could be too involved. But the particular effect of mediator depends on many circumstances i.e., hormone concentration and the receptor type. It may also undergo contraregulation. The majority of these hor- mones play an important role in the pathogenesis of CVS diseases', which can result in the development of new medicines [13].

\section{Conclusion}

Metabolic syndrome may be the main or associated cause of reproductive failure and cardiovascular disorders, but the possible interactions that exist between the systems must be focused on each patient individually.

\section{Conflict of Interest}

The author declares that there is no conflict of interest regarding the publication of this paper.

\section{References}

1. Morrison CD, Brannigan RE (2015) Metabolic syndrome and infertility in men. Best Pract Res Clin Obstet Gynaecol 29(4): 507-515.

2. Anderson RJ, Bahn GD, Moritz TE, Kaufman D, Abraira C, et al. (2011) Blood pressure and cardiovascular disease risk in the veterans affairs diabetes trial. Diabetes Care 34(1): 34-83.

3. Chow CK, Teo KK, Rangarajan S, Islam S, Gupta R, et al. (2013) PURE (Prospective Urban Rural Epidemiology) study investigators. Prevalence, awareness, treatment, and control of hypertension in rural and urban communities in high-, middle-, and low-income countries. JAMA 310(9): 959-368.

4. Traish AM, Abdou R, Kypreos KE (2009) Androgen deficiency and atherosclerosis: The lipid link. Vascul Pharmacol 51(5-6): 303-313.

5. Ramírez-Torres MA, Carrera A, Zambrana M (2000) High incidence of hyperestrogenemia and dyslipidemia in a group of infertile men. Ginecol Obstet Mex 68: 224-229.

6. Davoodi G, Amirezadegan A, Borumand MA, Dehkori MR, Kazemisaeid A, et al. (2007) The relationship between level of androgenic hormones and coronary artery disease in men. Cardiovasc J Afr 18(6): 362-366.

7. Zeller T, Schnabel RB, Appelbaum S, Ojeda F, Berisha F, et al. (2018) Low testosterone levels are predictive for incident atrial fibrillation and ischaemic stroke in men, but protective in women -results from the FINRISK study. Eur J Prev Cardiol 25(11): 1133-1139.

8. Schianca GP, Fra GP, Brustia F, Bellan M, Pirovano A, et al. (2017) Testosterone plasma concentration is associated with insulin resistance in male hypertensive patients. Exp Clin Endocrinol Diabetes 125(3): 171-175.

9. Gutiérrez C, Lozano-Hernández R, Lozano C, Villavicencio A (2014) Tensión arterial y masa corporal en mujeres infértiles con síndrome de ovario poliquístico y su relación con el perfil hormonal. Rev Obstet Ginecol Venez 74(3): 170-176.

10. Lozano-Hernández R, Gualdrón J, Camejo M, Velasco J, Villavicencio A (2015) Markers of accessory glands and seminal parameters in infertile men with overweight and obesity. J Metabolic Synd 6: 229

11. Tyagi V, Scordo M, Yoon RS, Liporace FA, Greene LW (2017) Revisiting the role of testosterone: Are we missing something? Rev Urol 19(1): 1624.

12. Gonzales GF, Lozano-Hernández R, Gasco M, Gonzales-Castañeda C Tapia V (2012) Resistance of sperm motility to serum testosterone in men with excessive erythrocytosis at high altitude. Horm Metab Res 44(13): 987-992.

13. Lacka K, Czyzyk A (2008) Hormones and the cardiovascular system. Endokrynol Pol 59(5): 420-432. 
(c) (i) Creative Commons Attribution 4.0 International License

For possible submissions Click Here

Submit Article

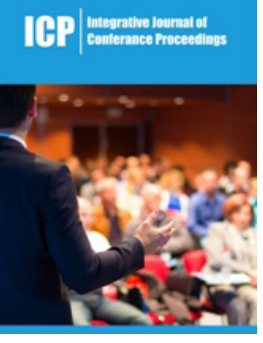

Integrative Journal of Conference Proceedings

\section{Benefits of Publishing with us}

- High-level peer review and editorial services

- Freely accessible online immediately upon publication

- Authors retain the copyright to their work

- Licensing it under a Creative Commons license

- Visibility through different online platforms 